\title{
Références bibliographiques du dossier : « La formation des élites »
}

\section{Bernadette Plumelle}

\section{OpenEdition}

1 Journals

Édition électronique

URL : https://journals.openedition.org/ries/1312

DOI : 10.4000/ries. 1312

ISSN : 2261-4265

Éditeur

France Education international

\section{Édition imprimée}

Date de publication : 1 septembre 2005

Pagination : 123-134

ISBN : 978-2-85420-564-0

ISSN : 1254-4590

Référence électronique

Bernadette Plumelle, "Références bibliographiques du dossier : "La formation des élites » », Revue internationale d'éducation de Sèvres [En ligne], 39 | septembre 2005, mis en ligne le 17 novembre 2011 , consulté le 21 septembre 2021. URL : http://journals.openedition.org/ries/1312 ; DOI : https://doi.org/ $10.4000 /$ ries. 1312 


\section{Références \\ bibliographiques}

\section{Bernadette Plumelle}

La formation des élites est un élément important du débat sur les finalités de l'école et semble devenir un enjeu social et politique. Les publications sur ce sujet ont été très nombreuses ces dernières années.

Cette bibliographie, non exhaustive, recense des documents référencés majoritairement dans la base de données du CIEP et concerne des travaux produits ces dix dernières années à l'exception de textes de référence. En introduction, le cadre général de l'étude est dressé; il recense une sélection de travaux sur les politiques éducatives face aux inégalités sociales. Une deuxième partie porte sur la formation des élites en France, objet d'un grand nombre de travaux: monographies, rapports et études statistiques. Des études comparatives et des monographies analysent ensuite la formation des élites en Europe et dans le monde.

Bibliographie arrêtée le 26 juin 2005.

\section{Cadre général}

BACHRACH Peter, The Theory of Democratic Elitism. A Critique, Boston: Little Brown and Compagny, 1967.

BAUER Michel, Des élites légitimes? Dossier, Problèmes politiques et sociaux, décembre 2000, $n^{\circ} 848,84 p$.

Ce dossier est constitué d'une sélection d'extraits d'articles et d'ouvrages; il est précédé d'une introduction de Michel Bauer. Le dossier est centré sur les élites politiques, économiques et administratives françaises; certains articles décrivent également ce que sont les élites à l'étranger. Les documents sélectionnés envisagent successivement les questions de la formation de ces groupes dirigeants, de leur mode de renouvellement, du sens de leur action et de leur rapport à la loi. En conclusion, les auteurs s'interrogent sur l'influence de la société de l'information dans l'émergence d'élites nouvelles.

BIRNBAUM Pierre, Les sommets de l'État: essai sur l'élite du pouvoir en France, Paris: Seuil, 1994, Points (première édition: 1977).

Institution prestigieuse attirant vers elle les élites de la nation issues des grandes écoles, l'État organise les activités les plus diverses grâce à ses fonctionnaires fidèles à la logique de leur rôle et aux valeurs du service public. Les élites politiques et celles de l'État en viennent alors souvent à se confondre mais de nouveaux processus de circulation des élites se profilent, estompant peu à peu les frontières autrefois si nettement défendues de l'État.

BOTTOMORE Tom B., Élites et sociétés, Paris: Stock, 1967, trad. fr. $1^{\text {re }}$ édition anglaise, 1964.

BUSINO Giovanni, Élites et élitisme, PUF/Paris, 1992, 128 p. (Que sais je?). 
COENEN-HUTHER Jacques, Sociologie des élites, Paris: Armand Colin, 2004, 172 p., bibliogr. (Cursus. Sociologie).

Comment définir les élites? Parle t-on de l'élite ou des élites? Comment se forment-elles? Constituent-elles de véritables classes dirigeantes? La diversité des approches et des réponses possibles permet à l'auteur d'apporter un éclairage sur un ensemble de questions: l'élite comme classe dirigeante, les élites comme groupes d'influences, la formation, le recrutement et la circulation des élites.

DOGAN Mattei ed., SCHEUCH Erwin K., CAMP Roderic A. and al., Special issue on elites, Comparative Sociology, janvier 2003, vol. 2, $n^{\circ} 1,289$ p. téléchargées du site http://www.brill.nl, bibliogr.

Le numéro spécial de cette revue offre une approche théorique des élites: hommes politiques, directeurs d'entreprises, détenteurs de capitaux, hauts fonctionnaires, leaders syndicaux, hauts gradés et grands intellectuels. L'article introductif s'intéresse aux liens entre les différentes catégories d'élites à l'échelle nationale et propose des comparaisons binaires entre la classe dirigeante française et celle de cinq pays: États-Unis, Grande-Bretagne, Italie, Allemagne et Japon. Puis chaque contribution donne un éclairage approfondi sur la situation et l'évolution des élites dans quelques pays - Allemagne, France, Mexique, Grande Bretagne, Canada - et dans deux zones géographiques - Asie du sud-est, Europe centrale et orientale.

Élites in R. BOUDON, F. BOURRICAUD, Dictionnaire critique de la sociologie, PARIS: Presses universitaires de France, 1994, p. 225-232.

KELLER Suzanne, Beyond the Ruling Class. Strategic Elites in Modern Society,

New York: Random House, 1963.

LASCH Christopher, La révolte des élites et la trahison de la démocratie, Paris: Climats, 1996, trad. fr. $1^{\text {re }}$ édition américaine, 1995, 270 p. (Sisyphe).

MERLLIÉ Dominique, PRÉVOT Jean, La mobilité sociale, Paris: La Découverte, 1997, 123 p. (Repères, $n^{\circ}$ 99).

Les auteurs présentent des éléments de problématique et de vocabulaire autour de la notion de mobilité sociale suivi d'un examen des outils d'observation. Les résultats d'enquêtes menées en France sont ensuite exposés pour dégager l'évolution des grands groupes sociaux et l'ouvrage se termine sur les rapports des destinées sociales avec le système éducatif.

PARETO Vilfredo, Traité de sociologie générale, Euvres complètes, éditées par Giovanni Busino, tome XII, Genève: Droz, 1968 (1 $1^{\text {re }}$ édition italienne, 1916).

SCOTT John, ed., The Sociology of Elites. vol.1: The Study of Elites, vol. 2: Critical Perspectives; vol. 3: Interlocking Dictatorships and Corporate Networks, Aldershot: Edward Elgar Publishing Ltd, 1990.

TURNER R. H. Sponsored and Contest Mobility in the School System (1960) in FORQUIN Jean-Claude Les sociologues de l'éducation américains et britanniques: Présentation et choix de textes, Paris: INRP, Bruxelles: De Boeck Université, 1997, 390 p., bibliogr., index (Portefeuille).

VEBLEN Thorstein, Théorie de la classe de loisir, Paris: Gallimard, 1979, trad. fr. $1^{\text {re }}$ éd. américaine, 1899. (Tel). 


\section{Politiques éducatives et inégalités sociales}

BOUDON Raymond, BULLE Nathalie, CHERKAOUI Mohamed dir., École et société: les paradoxes de la démocratie, Paris: PUF, 2001, 297 p., (Sociologies).

Les douze textes réunis dans cet ouvrage présentent les réflexions et analyses de philosophes et sociologues exprimées lors d'un colloque en 1999 à l'Université de Paris-Sorbonne. Ils s'articulent en deux grandes parties: la première est composée d'analyses des relations de l'éducation aux rapports sociaux en démocratie; la deuxième propose un ensemble de diagnostics et d'interprétations sur l'évolution contemporaine de l'inégalité des chances scolaires et de l'efficience des systèmes éducatifs.

BOURDIEU Pierre, Les Héritiers: les étudiants et la culture, Paris: Les Éditions de Minuit, 1964, 192 p., (Le Sens commun).

À partir des statistiques qui mesurent l'inégalité des chances d'accès à l'enseignement supérieur selon l'origine sociale et le sexe et en s'appuyant sur l'étude empirique des attitudes des étudiants et de professeurs ainsi que sur l'analyse des règles du jeu universitaire, les auteurs mettent en évidence, par-delà l'influence des inégalités économiques, le rôle de l'héritage culturel.

DURU-BELLAT Marie, Inégalités sociales à l'école et politiques éducatives, Paris: UNESCO/IIPE, 2003, 95 p., bibliogr. (Principes de la planification de l'éducation).

Dans les années 1960 et 1970, le thème des inégalités a dominé le débat sur l'éducation dans les pays industrialisés et des études ont montré le poids dominant de l'origine socio-économique dans les inégalités scolaires. Les travaux sur les inégalités qualitatives (orientation vers des filières plus ou moins prestigieuses) dominent la recherche contemporaine; l'auteur fait le point sur ces recherches et en donne une synthèse complète.

MAURIN Éric, Le ghetto français: enquête sur le séparatisme social, Paris: Seuil, 2004, 96 p. (La République des idées).

À partir de l'enquête annuelle sur l'emploi de l'INSEE, l'auteur a mesuré la constitution des différents voisinages de l'enquête et leur évolution dans le temps. Le choix de résidences et les stratégies mises en œuvre pour éviter certains quartiers ou villes sont fortement déterminés par les conditions de scolarité et de socialisation des enfants et des adolescents.

PERRENOUD Philippe, La fabrication de l'excellence scolaire: du curriculum aux pratiques d'évaluation. Vers une analyse de la réussite de l'échec et des inégalités comme réalités construites par le système scolaire, Genève: Droz, 1995, 348 p.

Ce livre s'inscrit dans le champ de la sociologie de l'éducation en proposant une introduction à la sociologie de l'évaluation scolaire et du curriculum. L'auteur a pour objet l'évaluation en milieu scolaire comme "procédé de fabrication de jugements d'excellence». S'appuyant sur plusieurs années d'observation dans les classes, il décrit l'analyse des programmes et des règles gouvernant l'évaluation mais aussi le travail scolaire et les jugements portés sur lui.

VAN ZANTEN Agnès, L'école de la périphérie, Paris: PUF, 2001, 424 p. (Le lien social).

La tendance à la différenciation sociale, ethnique et scolaire des élèves des établissements s'est accentuée depuis les années 1980. Dans cet ouvrage, l'auteur analyse les processus multiples 
qui mènent aux ségrégations scolaires. Cette étude ne s'intéresse pas seulement aux écoles de banlieue; elle apporte aussi un autre regard sur la scolarisation et le rôle des dynamiques locales dans la reproduction et la transformation des systèmes scolaires.

VAN ZANTEN Agnès, Les politiques d'éducation, Paris: PUF, 2004, 126 p. (Que sais-je?).

Cet ouvrage donne une grille de lecture des politiques éducatives et de leur mise en pratique. La première partie du livre débute par une présentation synthétique des approches, des modèles et des démarches qui ont été développées pour étudier les politiques d'éducation. L'analyse de ces politiques en France commence par l'étude des valeurs et des idées qui orientent les choix éducatifs, de leurs traductions politiques. La seconde partie examine le degré d'autonomie des dirigeants nationaux, les instances et les modes de négociation ainsi que le fonctionnement de l'administration.

\section{La formation des élites en France}

ADANGNIKOU Noël, PAUL Jean-Jacques, Efficience de l'enseignement supérieur dans la production des élites: le cas des classes préparatoires scientifiques aux grandes écoles. Rapport pour le Commissariat Général du Plan, Les Cahiers de l'IREDU, $n^{\circ}$ 67, octobre 2004, 164 p.

La filière des classes préparatoires contribue-t-elle à produire des compétences spécifiques justifiant les moyens très importants alloués par l'État? Cela permet-il aux élèves issus de ces filières une carrière spécifique en terme de rémunération ou d'emploi? Après une approche générale des classes préparatoires aux grandes écoles, cette étude expose les outils de mesure de l'efficacité interne ainsi que les résultats de l'enquête menée auprès d'élèves en première année. La dernière partie de l'étude porte sur les analyses effectuées à partir de données produites par d'autres organismes.

AGHION Philippe, COHEN Elie, Éducation et croissance, Paris: Documentation française, 2004, 143 p.

Le rapport dénonce une organisation de l'enseignement supérieur marquée par une coupure entre les grandes écoles dotées de moyens financiers importants et les universités, parents pauvres du système. À partir de l'enquête PISA, il apparaît que ses performances dans le secondaire sont dans la moyenne des partenaires de l'OCDE, que la massification et la démocratisation des universités donnent nombre d'échecs en DEUG et que les universités pâtissent d'une faible sélection. Le rapport présente par ailleurs les spécificités et l'évolution du modèle universitaire français et propose trois scénarios d'évolution de l'université en précisant les principaux leviers de la réforme.

\section{ALBOUY Valérie, WANECQ Thomas, Les inégalités sociales d'accès aux grandes écoles, Économie et statistique, 2003, $n^{\circ} 361$, p. 27-52.}

L'analyse, portant sur l'origine sociale des élèves ayant accédé aux grandes écoles entre 1940 et 1980, souligne la permanence d'une sélection sociale et culturelle très marquée. Une accentuation des inégalités d'accès aux grandes écoles se note dans les années 1980 due au renforcement de leur sélectivité et à la plus grande ouverture des troisièmes cycles universitaires à caractère professionnalisant. 
ATTALI Jacques dir., Pour un modèle européen d'enseignement supérieur: rapport au Ministre de l'Éducation nationale, de la recherche et de la technologie, Paris: Ministère de l'éducation nationale, 1998, 77 p. téléchargées du site http://www.education.gouv.fr/

Ce rapport, coordonné par Jacques Attali, est le résultat d'une consultation d'universitaires, de chercheurs et de chefs d'entreprise, sur le système d'enseignement supérieur français. La première mission des universités et des grandes écoles n'est plus le recrutement des cadres de l'État. Le rapport préconise de nouvelles missions, de nouveaux principes d'organisation à l'enseignement supérieur. Les grandes écoles devront, pour continuer de former un vivier de très haut niveau, développer leurs activités de recherche et s'ouvrir davantage aux étudiants venus de l'enseignement technique et de l'étranger. Des synthèses des réformes en cours dans d'autres pays (Allemagne, Italie, Royaume-Uni, États-Unis) sont présentées ainsi que, en annexe, des statistiques sur l'état de l'enseignement supérieur en France.

BAUDELOT Christian, MATONTI Frédérique, Le recrutement social des normaliens 1914-1992, in École normale supérieure: le livre du bicentenaire, Paris: PUF, 1994, p. 155-190.

Les auteurs retracent l'évolution des origines sociales des élèves de l'École normale supérieure (ENS) depuis 1914, décennie après décennie et esquissent un portrait social des élèves normaliens d'aujourd'hui. Ils établissent aussi les similitudes et différences avec le recrutement de l'École polytechnique et de l'École nationale d'administration.

BAUDELOT Christian, FAUROUX Roger, CALAN Dominique de, Réformer les grandes écoles, Le Monde de l'éducation, janvier 1998, p. 82-86.

BAUER Michel, BERTIN-MOUROT Bénédicte, La triple exception française. À propos de la formation des élites, Esprit, octobre 1997, p. 47-59.

L'article distingue trois traits caractéristiques à la formation des élites en France: le poids du diplôme initial, le haut niveau de responsabilité dès le premier poste et l'importance de la fuite vers le privé. Les auteurs considèrent ce modèle d'élitisme français comme un modèle en crise et proposent une autre construction de l'autorité légitime qui ne soit plus fondée sur le seul mérite scolaire.

BOUHIA Rachid, Les étudiants en classes préparatoires aux grandes écoles. Année 2003-2004, Note d'information - DEP, $n^{\circ}$ 04.16, juin 2004, 6 p.

Les classes préparatoires aux grandes écoles (CPGE) accueillaient en 2003-2004 72000 étudiants et $8,9 \%$ des bacheliers généraux et technologiques de la session 2003. Les effectifs sont stables mais leurs évolutions sont différentes selon les filières et l'année d'étude.

BOURDIEU Pierre, La noblesse d'État: grandes écoles et esprit de corps, Paris: Éditions de Minuit, 1989, 568 p., (Le sens commun).

Comment s'est constituée la configuration singulière de pouvoirs, intellectuels, politiques, économiques qui dominent les sociétés contemporaines? Comment ces pouvoirs obtiennentils notre reconnaissance? Pierre Bourdieu mobilise toutes les ressources de la statistique, de la théorie sociologique et de l'histoire sociale pour porter au jour les structures les plus profondes des systèmes d'enseignement et du travail de consécration accompli par l'institution scolaire, notamment à travers les grandes écoles. 
CHARPAK Georges, BAUDELOT Christian, VERGNAUD Gérard, et al., Formation des élites et école pour tous, Ministère de l'éducation nationale de l'enseignement supérieur et de la recherche/France, 1996, 213 p., ill., photos.

Au cours de leur journée d'étude de 1995, les inspecteurs généraux de l'Éducation nationale ont débattu de ce problème inhérent aux systèmes éducatifs des sociétés développées: comment démocratiser l'enseignement et réduire l'échec scolaire tout en préservant la formation d'une élite. Les diverses contributions ont tenté d'apporter, sinon des réponses, du moins des explications, dans des champs aussi variés que la physiologie, la neurobiologie, la sociologie, la psychologie.

\section{CROZIER Michel, TILLIETTE Bruno, La crise de l'intelligence: essai sur l'im-} puissance des élites à se réformer, Paris: Interéditions, 1995, 200 p.

Publié avec la collaboration du journaliste Bruno Tilliette, l'ouvrage de Michel Crozier a pour point de départ le «désastre du système de décision français». La crise de la France actuelle est, selon les auteurs, avant tout morale et intellectuelle: elle a perdu confiance dans des élites qui s'avèrent incapables de réformes. Ces réformes sont cependant possibles à condition de s'appuyer sur une véritable écoute des acteurs concernés, permettant de reconnaître leurs problèmes et de transformer en même temps les mentalités et le système.

\section{DEP: Direction de l'évaluation et de la prospective/France, Les classes prépara-} toires aux grandes écoles: évolutions sur vingt-cinq ans, Les dossiers, $n^{\circ} 146$, décembre 2003.

Dans le cadre d'un colloque organisé en mai 2003 sur le thème «Démocratie, classes préparatoires et grandes écoles", la Direction de l'évaluation et de la prospective (DEP) a réalisé un dossier rassemblant informations statistiques et études sur les classes préparatoires. Le premier document est l'exposé présenté lors du colloque montrant l'évolution des classes préparatoires et les caractéristiques de la population qu'elles accueillent; il est suivi d'un article qui analyse les flux migratoires des bacheliers à l'entrée des classes préparatoires et met en évidence les disparités existant selon les types de classes et le sexe. La troisième partie rassemble les Notes d’information publiées par la DEP sur ce sujet.

DUBET François, DURU-BELLAT Marie, L'hypocrisie scolaire. Pour un collège enfin démocratique, Paris: Seuil, 2000, 236 p., (L'épreuve des faits).

Les auteurs dressent le bilan des connaissances sur le collège et proposent de rompre avec l'image d'un établissement tiraillé dans ses contradictions: à la fois l'héritier de l'ancien lycée de l'excellence et l'école de tout le monde. Ils prônent la nécessité de rendre le collège moins injuste, plus efficace et plus vivable pour les élèves et les enseignants.

DURU-BELLAT Marie, KIEFFER Annick, ADANGNIKOU Noël, Efficacité et équité de l'enseignement supérieur français dans la production des élites, in FELOUZIS Georges dir., Les mutations actuelles de l'Université, Paris: Presses Universitaires de France, 2003.

\section{EURIAT Michel, THELOT Claude, Le recrutement social de l'élite scolaire en} France: évolution des inégalités de 1950 à 1990, Revue française de sociologie, septembre 1995, vol. XXXVI, $n^{\circ} 3$; p. 403-438, bibliogr.

L'article est consacré à l'évolution au cours des dernières décennies du recrutement des élèves des grandes écoles parmi les plus prestigieuses: l'École polytechnique, l'École normale supérieure, l'ENA et HEC. L'auteur cherche à cerner la capacité du système d'enseignement à faire accéder les couches populaires et moyennes à l'élite scolaire; après avoir retracé l'évolution générale des inégalités devant l'école, il montre que ces écoles se sont, non pas fermées aux couches populaires, mais moins ouvertes que le reste du système éducatif. 
FAUCONNIER Patrick, La fabrique des meilleurs: enquête sur une culture d'exclusion, Paris: Seuil, 2005, 288 p. (L'histoire immédiate).

L'auteur, journaliste, analyse la société française comme une société élitiste où la performance du «meilleur» commande et verrouille l'accès à l'emploi. Le système d'enseignement sélectionne très tôt, sur la base des savoirs théoriques, l'élite de la nation et lui réserve les meilleures filières et les postes clés. Pour l'auteur, qui s'appuie sur une comparaison entre les systèmes éducatifs français et américain, cette pratique brime les autres formes d'intelligence: sens du commerce, talent manuel, sens artistique et imagination.

FERRAND Michèle, IMBERT Françoise, MARRY Catherine, Excellence scolaire: une affaire de famille. Le cas des normaliennes et normaliens scientifiques, L'Harmattan/Paris, 1999, 210 p. (Bibliothèque de l'éducation).

En l'espace de deux ou trois décennies, les filles ont réussi une percée spectaculaire dans le domaine des études supérieures mais celles qui réussissent à entrer à l'École normale supérieure dans les disciplines scientifiques comme les mathématiques et la physique restent l'exception. Trois chercheurs ont réalisé une enquête auprès des normaliens et normaliennes scientifiques des promotions 1985 à 1990 pour déterminer les stratégies éducatives des familles des normaliennes et ont mis en évidence les conditions personnelles et familiales qui ont autorisé ces destinées improbables.

\section{LAZUECH Gilles, Le processus d'internationalisation des grandes écoles fran-} çaises, Actes de la recherche en sciences sociales, mars 1998, $n^{\circ}$ 121-122, p. 66-76.

L'auteur s'appuie sur l'exemple des grandes écoles françaises de commerce et d'ingénieurs pour montrer comment se produit un processus d'internationalisation et les conséquences qu'il engendre au sein de l'espace national. L'internationalisation avive la concurrence entre les écoles et contribue à produire des effets de recomposition dans le champ de la formation des cadres.

LEMAIRE Sylvie, Profil et devenir des élèves inscrits dans une classe préparatoire aux grandes écoles, Note d'information - DEP, $n^{\circ}$ 01.31, juin 2001, 6 p.

Les bacheliers qui intègrent une classe préparatoire présentent des caractéristiques communes très fortes et ont suivi un parcours scolaire sans faute mais leurs motivations diffèrent selon le type de classe; l'intérêt pour les disciplines enseignées est déterminant pour ceux qui intègrent une classe littéraire tandis que les débouchés professionnels sont déterminants pour les bacheliers scientifiques. Le devenir des élèves est également très différent selon les spécialités.

\section{LEOTARD Marie-Laure de, Le dressage des élites. De la maternelle aux grandes écoles: un parcours pour initiés, Paris: Plon, 2001, 242 p.}

L'auteur, journaliste, dénonce les mécanismes de sélection en jeu dans le système éducatif français dès le plus jeune âge. Son ouvrage, émaillé d'exemples, suit les différents niveaux scolaires: collège, lycée, classes préparatoires et s'attarde sur trois grandes écoles: HEC, Polytechnique et l'ENA.

LEWANDOWSKI Olgierd, Différenciation et mécanismes d'intégration de la classe dirigeante. L'image sociale de l'élite d'après le Who's Who in France, Revue française de Sociologie, $n^{\circ}$ 1, 1974, p. 43-73.

MAGLIULO B., Les Grandes écoles, Paris: PUF, 1982 (Que sais-je?). 
MUXEL Anne dir., Les étudiants de Sciences Po. Leurs idées, leurs valeurs, leurs cultures politiques, Paris: Presses de Sciences Po, 2004, 269 p.

En janvier 2002, une équipe de chercheurs du Centre de recherches politiques de Sciences Po (CEVIFOP) a organisé une enquête auprès de 2450 étudiants de l'Institut d'études politiques (IEP) de Paris. Les auteurs de cette enquête ont voulu cerner leurs valeurs, idées et cultures politiques. L'ouvrage permet de mettre en lumière certains éléments d'une culture politique commune à l'école à travers des caractéristiques socio-politiques diversifiées.

PONCET François dir., BRACONNIER Alain dir., Classes préparatoires: des étudiants pas comme les autres, Paris: Bayard, 149 p., (Collection Païdos).

Les élèves des classes préparatoires et des grandes écoles apparaissent comme privilégiés mais la pression à laquelle ils sont soumis peut faire apparaître des difficultés psychologiques souvent mal reconnues et analysées. L'ouvrage réunit des contributions d'une dizaine de spécialistes, de la psychanalyse à la sociologie, pour analyser ce phénomène et déterminer dans quelle mesure un certain déséquilibre est constitutif des conditions de réussite à ce type de concours.

ROSANVALLON Pierre, Les élites françaises, la démocratie et l'État, entretien avec Pierre Rosanvallon, mené par Olivier Mongin et Lucile Schmid, Esprit, $n^{\circ} 10$, octobre 1997, p. 60-72.

SILGUY Yves-Thibault de, Moderniser l'État: l'encadrement supérieur. Rapport au ministre de la fonction publique, de la réforme de l'État et de l'aménagement du territoire, Paris: La Documentation française, décembre 2003, 137 p. La commission sur la réforme de l'ENA (École nationale d'administration) et la formation des cadres supérieurs de la fonction publique, présidée par Yves-Thibault de Silguy, s'attache tout d'abord à définir les besoins en terme de recrutement et cherche à en déduire, pour les vingt prochaines années, les compétences susceptibles d'être exigées des hauts fonctionnaires. Elle procède ensuite à une analyse des données portant sur l'organisation, la gestion, la formation et la mobilité de l'encadrement supérieur de la fonction publique. Sur la base de ce bilan, la Commission présente ses propositions d'actions qui portent, entre autres, sur une amélioration des conditions de recrutement et un rééquilibrage entre formation initiale et formation permanente.

SULEIMAN Ezra N., Les Élites en France. Grands corps et grandes écoles, Paris:

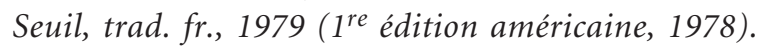

TENZER Nicolas, DELACROIX Rodolphe, LOTTAUX Philippe, Les Élites et la fin de la démocratie française, PUF/Paris, 1992, 232 p., (Politique d'aujourd'hui).

La France est un pays de tradition élitiste. Or les auteurs diagnostiquent une crise au sommet de la hiérarchie sociale; ils en analysent les causes et les effets et prescrivent les remèdes. Ils dénoncent vigoureusement un système médiatique qui favorise l'émergence de fausses élites et mettent en cause l'élite administrative, dont ils contestent les filières de formation. Ce livre émet des propositions concrètes, notamment sur la formation et les modes de construction des carrières et propose de repenser en profondeur les systèmes d'éducation et de sélection avec un renforcement de la culture générale à la base des enseignements, de souplesse dans les cursus et de diversification dans les recrutements.

TENZER Nicolas, À quoi l'ENA doit-elle servir?, Esprit, octobre 1997, p. 19-32. Comment définir ce qu'on attend des fonctionnaires d'encadrement et de direction, comment les sélectionner et les former? L'auteur revient sur les origines de cette institution et sur la formation délivrée et apporte un certain nombre de propositions sur les principes qui doivent déterminer la formation délivrée à l'ENA. 
VAN ZANTEN Agnès coord., L'école: l'état des savoirs, Paris: La Découverte, 20004, 420 p. (Textes à l'appui. Série l'état des savoirs).

Cet ouvrage collectif, rassemblant près d'une cinquantaine de contributions, présente un état des savoirs sur la situation de l'école française. Les travaux produits sont regroupés en six rubriques: un état des lieux du système d'enseignement, les politiques éducatives et leur évolution, les savoirs que l'école doit transmettre, les acteurs, les parcours (analyse de la diversité des trajectoires scolaires suivant le sexe, l'appartenance sociale ou ethnique) et les enjeux.

WAGNER Anne-Catherine, Les nouvelles élites de la mondialisation: une immigration dorée en France, Paris: Presses universitaires de France, 1998, 236 p., bibliogr., (Sciences sociales et sociétés).

Une partie croissante de l'immigration en France est le fait de cadres supérieurs. Ce livre présente les résultats d'une enquête sur les étrangers de haut niveau social et révèle la formation d'un groupe nouveau. Une première présentation s'attache à dégager les principes d'un statut privilégié au regard des immigrés «ordinaires». Il sera suivi d'une étude des écoles destinées aux enfants de cadres internationaux et d'une analyse sur les processus de transmission familiale.

\title{
Formation des élites en Europe et dans le monde
}

\section{Études transnationales}

\begin{abstract}
BROADY D. éd., CHAMTKO N. éd., SAINT MARTIN de M. éd., Formation des élites et culture transnationale - Colloque de Moscou 27-29 avril 1996, Paris/Uppsala: CSEC-EHESS \& SEC, ILLI, Université d'Uppsala, 1997, 363 p.

Ce colloque, tenu à Moscou en avril 1996, a confronté des études sur la formation des élites dans différentes zones géographiques. Les premières, menées en Russie, analysent la constitution des nouvelles élites et la reconversion des anciens groupes dirigeants. La deuxième partie présente des études sur les transformations des modes de formation des élites en Europe et en Afrique. Dans la troisième partie ont été réunies les contributions qui traitent des processus d'échanges de produits culturels et scientifiques.
\end{abstract}

OCDE: Organisation de coopération et de développement économiques/Paris, International mobility for the highly skilled, Paris: OCDE, 2002, 348 p., statistiques, bibliogr., annexes.

Face à une pénurie nationale, de plus en plus de pays mettent en place des mesures visant à faciliter le recrutement de travailleurs qualifiés étrangers. Dans ce contexte, l'OCDE a organisé à Paris les 11 et 12 juin 2001 le séminaire «Mobilité internationale des travailleurs les plus qualifiés: de l'analyse statistique à la formulation de politiques». La première partie regroupe définitions et mesures de cette population et de ce flux. À travers des études de cas de pays membres de l'OCDE et extérieurs à l'Organisation, le second chapitre traite des tendances de cette mobilité internationale spécifique et de son impact économique. Enfin, la troisième partie décrit comment les politiques, en matière de migration et de sciences et technologie, peuvent influencer cette mobilité. 
OCDE: Organisation de coopération et de développement économiques, Regards sur l'éducation: les indicateurs de l'OCDE 2004, Paris: OCDE, 2004, 497 p., annexes, glossaire, statistiques.

Les indicateurs de l'OCDE mesurent l'état actuel de l'éducation à l'échelle mondiale. Ce rapport, organisé thématiquement, présente un ensemble d'indicateurs actualisés et comparables sur les systèmes d'enseignement. Ceux-ci permettent d'examiner les problèmes d'équité liés aux résultats de l'enseignement et à l'accès à la formation à partir de critères d'âge, de sexe, de milieu socio-économique, de type d'établissement et de domaine d'études.

\section{Europe et États-Unis}

GOLDRING Maurice, Voie royale, voie républicaine: formation des élites en France et en Grande-Bretagne, Paris: Éd. Syllepse, 2000, 126 p. bibliogr. (Utopie critique).

Les systèmes d'enseignement français et anglais se sont construits autour de valeurs dissemblables, méritocratie ou aristocratie. En comparant les deux systèmes, l'auteur s'attache à mettre à jour les dispositifs de sélection. Il décrit d'abord les origines des grandes écoles et des public schools, puis établit une comparaison dans la sélection des élites. La dernière partie porte sur les réformes menées dans les deux pays pour répondre aux critiques de ces systèmes de formation profondément inégalitaires.

KARABEL Jérome, Harvard et le critère du mérite, Actes de la recherche en sciences sociales, décembre 2000, $n^{\circ} 135$, p. 63-75.

L'auteur dresse un panorama des principes et des actions menées par le recteur de Harvard, James Bryant Conant, de 1934 à 1953. Celui-ci a considéré que les aptitudes des étudiants devaient décider de l'accès à l'enseignement supérieur, et non les privilèges héréditaires, mais sa vision reste très élitiste. Ses écrits ont exercé, plus généralement, une grande influence sur la structure et les pratiques des écoles et des universités américaines.

HARTMANN M., Class-specific habitus and the social reproduction of the business elite in Germany and France, Sociological Review, vol. 48, $n^{\circ} 2,2000$, p. 241-261. [PDF]

Fondé sur deux études empiriques des cadres supérieurs, cet article examine les aspects principaux de la théorie de Pierre Bourdieu sur la reproduction sociale. À cet effet ont été étudiées l'origine sociale et la trajectoire éducative des présidents des cent plus grandes entreprises allemandes et françaises de 1995, comparées aux statistiques correspondantes des années 1970 à 1972. Dans les deux pays, presque 80\% de cadres supérieurs sont recrutés à l'intérieur de l'élite sociale même si les mécanismes de sélection diffèrent en France et en Allemagne.

PANAYOTOPOULOS Nikos, Les "grandes écoles" d'un petit pays. Les études à l'étranger: le cas de la Grèce, Actes de la recherche en sciences sociales, mars 1998, $n^{\circ}$ 121-122, p. 77-91.

La Grèce se range au premier rang des pays dont les étudiants réalisent leur cursus universitaire à l'étranger. Le passage par les établissements étrangers tend à commander l'accès aux postes prestigieux, tandis que les établissements nationaux se limitent le plus souvent à des formations pour des postes d'exécution, de techniciens, de cadres moyens. 
PERSELL Caroline H., COOKSON Peter W., Pensionnats d'élite. Ethnographie d'une transmission de pouvoir, Actes de la recherche en sciences sociales, juin 2001, $n^{\circ} 138$, p. 56-65.

Bien qu'ils accueillent moins de $1 \%$ des lycéens, les internats privés américains forment un nombre disproportionnellement élevé de personnalités marquantes dans le monde des affaires, de la politique et de la culture. Traditionnels et conservateurs, les lycées privés constituent une institution typiquement américaine sans d'équivalent; ils ont joué un rôle important dans la formation et le maintien d'une classe dominante américaine.

SULEIMAN Erza, MENDRAS Henri dir., Le recrutement des élites en Europe, Paris: La Découverte, 1997, 264 p. (Recherches).

Cet ouvrage réunit un ensemble de contributions sur la formation des élites. Chaque pays d'Europe occidentale possède des filières de recrutement qui lui sont particulières et les auteurs dressent un panorama des institutions et des conceptions qui concourent au recrutement des élites politiques, administratives et économiques en France, Allemagne, GrandeBretagne, Italie et Espagne. L'analyse porte sur les mécanismes qui permettent aux principaux pays de l'Union européenne de constituer leur classe dirigeante et décrit aussi les milieux socio-professionnels d'où cette classe est issue. Enfin, deux exemples pris hors de l'Union européenne, la Pologne et les États-Unis sont proposés.

STANWORTH Philip, GIDDENS Anthony, Elites and Power in British society, Cambridge: Cambridge University Press, 1974.

\section{Amérique latine}

BERCHENKO Pablo, La formation des élites latino-américaines dans l'interaction des domaines anglo-hispaniques in Latinos et Yankees: Interactions dans l'espace anglo-hispanique des Amériques, Actes du troisième colloque du Centre d'analyse et des recherches nord-américaines et latines (CARNAL), Université de Provence, Aix-Marseille I, septembre 1997. Publications de l'Université de Provence, 1999, p. 43-59.

Les États-Unis et la formation des élites chiliennes (1945-1999) in VAGNOUX Isabelle, Les États-Unis et les élites latino-américaines, Actes du colloque des 24 et 25 septembre 1999 de l'institut de Recherche du monde anglophone. Publication de l'Université de Provence, 2000, p. 99-116.

DROSILA VASCONCELLOS Maria, L'internationalisation des écoles de gestion au Brésil, Actes de la recherche en sciences sociales, mars 1998, n 121-122, p. 62-65.

Une importante dimension de l'internationalisation des élites chargées des politiques macroéconomiques au Brésil est la formation académique reçue à l'étranger, en particulier aux ÉtatsUnis. L'article montre comment le crédit acquis à travers ces rapports internationaux est un des atouts mobilisés par cette élite dans les luttes internes au système politique. 


\section{Afrique}

INSTITUT FRANÇAIS DE RECHERCHE EN AFRIQUE, LEBEAU Yann ed., OGUNSANYA Mobolaji ed., The dilemma of post-colonial universities: elite formation and the restructuring of higher education in Sub-Saharan Africa = Formation des élites et restructuration de l'enseignement supérieur en Afrique subsaharienne, African BookBuilders-IFRA/Ibadan, 2000, VIII-334 p., ill., références bibliogr. Index.

Recueil d'articles présentés à la Conférence organisée par l'Institut français de recherche en Afrique, et l'Université d'Ibadan, 26-27 Octobre 1998.

VERMEREN Pierre, La formation des élites marocaines et tunisiennes: des nationalistes aux islamistes 1920-2000, Paris: La Découverte, 2002, 512 p., (Recherches).

Quel rôle a joué l'école dans la reproduction des élites au Maghreb au cours du XX $\mathrm{X}^{\mathrm{e}}$ siècle? L'auteur retrace cette histoire en brossant le portrait de quatre générations. Jusqu'aux indépendances en 1956, le baccalauréat est la clef de la conquête des études supérieures pour une infime minorité de musulmans. Les réformes de grande ampleur, mises en place à partir de 1945 et renforcées à l'indépendance, permettent l'émergence, du début des années soixante jusqu'au milieu des années soixante-dix, de la génération de l'ouverture. Dans le dernier quart du siècle, on assiste à un processus de consolidation de la nouvelle élite.

\section{Asie}

SABOURET Jean-François (dir.), La dynamique du Japon, éditions Saint-Simon, 2005.

YU Hu, La méritocratie et l'éducation en Chine, Pratiques de formation: analyses, 2003, $n^{\circ}$ 45-46, p. 51-65.

L'auteur examine la notion de méritocratie dans la Chine traditionnelle. Rappellant la vision de l'éducation confucéenne, il analyse ce qu'est devenue la méritocratie dans la Chine actuelle. 